Original Article

\title{
FUNGAL PROFILE OF INFECTIOUS KERATITIS IN A TERTIARY CARE HOSPITAL - OUR EXPERIENCE
}

\author{
Sanjeev H. ${ }^{1}$, Karnaker Vimal K. ${ }^{1}$, Pai Vijay ${ }^{2}$, Pai Asha K.B. ${ }^{1}$, Rai Rekha ${ }^{1} \&$ Krishnaprasad M.S. ${ }^{1}$ \\ ${ }^{1}$ Department of Microbiology \& ${ }^{2}$ Department of Ophthalmology, \\ K. S. Hegde Medical Academy, Nitte University, \\ Mangalore - 575 018, India. \\ Correspondence: \\ Dr. Sanjeev H., \\ Assistant Professor, Phone: +91824 2204490-92, \\ Mobile : 99722 12280, E-mail : drsanjeevh@gmail.com
}

\begin{abstract}
:
Infectious keratitis world wide are a leading cause of ocular morbidity and blindness. A large number of filamentous fungi, Yeasts and Zygomycetes have been incriminated as the causative agent of mycotic keratitis. Early diagnosis and treatment is important in preventing complications like corneal perforation, scleral spread and endopthalmitis. The present study was conducted to elucidate the epidemiological features of mycotic keratitis and study the fungal profile of mycotic keratitis of patients attending our hospital, which is situated on the coastal area of Karnataka.

A total of 127 patients with infectious keratitis were investigated between January 2009 to June 2010. Corneal scraping was obtained from 127 patients under aseptic precaution. The scraping was subjected to $10 \% \mathrm{KOH}$ wet mount, Gram's staining and culture.

Of the total 127 patients suspected of having infectious keratitis, 44 (34.65\%) were found to be positive for fungal aetiology. Of these, $40(90.90 \%)$ cases were positive on direct microscopy for fungal elements and $26(59.09 \%)$ cases showed growth on culture after incubation for 2-8 days. In $14(31.81 \%)$ cases, the culture was found to be sterile despite positive direct microscopic findings, but the results were consistent with clinical findings. Positive culture was obtained in $4(09.09 \%)$ cases where direct microscopy was found to be negative. The commonest fungi isolated were Aspergillus species (61.5\%)

M ycotic keratitis continues to be an important cause of ocular morbidity, predominantly among rural population. Prompt diagnosis and early institution of antifungal therapy may limit the ocular morbidity and the sequelae of infectious keratitis. As the manifestation of mycotic keratitis is often confusing, a high degree of suspicion with sound knowledge of predisposing factors and microbiological confirmation is very essential to initiate appropriate therapy.
\end{abstract}

Keywords: Keratitis, 10\% KOH mount, Aspergillus species.

\section{Introduction:}

Infectious keratitis world wide are a leading cause of ocular morbidity and blindness ${ }^{1}$. The incidence of mycotic keratitis is much higher in tropical and sub tropical region than in countries having temperate climate ${ }^{2}$. A large number of filamentous fungi, Yeasts and Zygomycetes have been incriminated as the causative agent of mycotic keratitis ${ }^{3}$. However, the etiological and epidemiological pattern of infectious keratitis varies significantly from place to place based on climatic, geographical and socioeconomic condition ${ }^{4}$. The risk factors for fungal keratitis can be grouped into 2 categories -1) factors that disrupt the corneal epithelium, ex; trauma by vegetative matter and 2 ) factors that impair the immunologic response to trauma like steroid and antibiotic abuse ${ }^{5}$.
In a developing country like India, with a large agrarian population, accidental corneal trauma with vegetative matter is very common. Confounding to the problem is the quite varied and often overlapping clinical features of fungal keratitis ${ }^{6}$.

Early diagnosis and treatment is important in preventing complications like corneal perforation, scleral spread and endopthalmitis?. Basing the diagnosis on clinical findings alone can lead to erroneous treatment. Therefore, gold standard in diagnosis remains direct examination and culture of corneal scraping ${ }^{8}$.

The present study was conducted to elucidate the epidemiological features of mycotic keratitis and study the fungal profile of mycotic keratitis of patients attending our 
hospital, which is situated on the coastal area of Karnataka.

\section{Materials and methods:}

The study was conducted in the Departments of Ophthalmology and Microbiology, K.S. Hegde Medical College \& Hospital, M angalore. A total of 127 patients with infectious keratitis were investigated between January 2009 to June 2010. Infectious keratitis was defined as stromal infiltration and/or suppuration, associated symptoms \& signs of inflammation with or without hypopyon or loss of corneal epithelium. Patients with microbiological evidence of keratitis due to bacteria, virus and Acanthamoeba species were excluded from this study?

In all these patients, a detailed ocular examination was carried out and corneal scraping from the leading edge and base of the ulcer was taken under all aseptic precaution by an experienced ophthalmologist. The samples were sent to Department of Microbiology immediately. Direct microscopic examination of the corneal scraping was performed with $10 \%$ potassium hydroxide $(\mathrm{KOH})$ wet mount and Gram's staining?.

At the same time scrapings were also inoculated directly on Non nutrient agar (NNA) with overlay of Escherichia coli, Chocolate agar and Sabourauds dextrose agar (SDA) without cycloheximide, in rows of C-shaped streaks. Chocolate agar and NNA were incubated aerobically at $37{ }^{\circ} \mathrm{C}$. They were examined daily and discarded after 7 days if no growth was seen. SDA was incubated at $25^{\circ} \mathrm{C}$ and $37^{\circ} \mathrm{C}$ for 4 weeks. The media was checked for any fungal growth daily during the $1^{\text {st }}$ week and twice a week for the subsequent 3 weeks. Any growth obtained was identified by standard fungal identification techniques ${ }^{10}$. If no growth was observed after 4 weeks of incubation, the media were labelled sterile and discarded ${ }^{9}$. The culture was considered positive if at least one of the following criteria was fulfilled-

\section{The growth of the same organism demonstrated on one} or more solid and/or if there was confluent growth at the site of inoculation on at least one medium.

2. The growth on one medium was consistent with direct microscopy

3. The same organism was grown at repeated corneal scrapping.

4. The growth was consistent with clinical findings.

\section{Results:}

Of the total 127 patients suspected of having infectious keratitis, 44 (34.65\%) were found to be positive for fungal aetiology. Of these, $40(90.90 \%)$ cases were positive on direct microscopy for fungal elements and $26(59.09 \%)$ cases showed growth on culture after incubation for 2-8 days. In 14 (31.81\%) cases, the culture was found to be sterile despite positive direct microscopic findings, but the results were consistent with clinical findings. Positive culture was obtained in $4(09.09 \%)$ cases where direct microscopy was found to be negative. (Table1). There were a total of 26 isolates, which included Aspergillus species 16(61.5\%), Fusarium species 3(11.53\%) Penicillium species $3(11.53 \%)$, Candida species $2(07.69 \%)$ and one isolate $(03.86 \%)$ each of M ucor species and Paecilomyces species. (Table-2)

Comparison of $10 \% \mathrm{KOH}$ mount and Gram stain showed sensitivities of $90.91 \%$ and $75 \%$ respectively. All the patients suspected to have infectious keratitis were aged between 20-61years. The most frequently affected age group was $41-50$ years with 41 (32.28\%) cases followed by $31-40$ years with $38(29.92 \%)$ cases. Men, $92(72.44 \%)$ cases, were more frequently affected than women, $35(27.55 \%)$ cases.

Most of the patients, $104(81.88 \%)$, were from a rural background and majority of them, 96/104(92.3\%) cases, were involved in farming. Corneal trauma by vegetative matter, 24/44(54.54\%) cases, such as paddy, wood splinters, thorns, was the most common predisposing factor. Corneal trauma, other than by vegetative matter, was documented in $4 / 44(9.09 \%)$ cases. The other risk factors elicited were long term use of topical steroid and/or antibiotics, 8/44(18.18\%) cases and chronic disease like diabetesmellitus, $2 / 44(4.54 \%)$ cases. 


\section{Discussion :}

Mycotic keratitis is much more frequent in developing countries, such as India, than in developed countries ${ }^{11}$. Various Indian literatures reported a prevalence of 7-40\% in different parts of India ${ }^{2}$. In the present study, the incidence was found to be $34.65 \%$. This is very much in accordance with a study reported from the similar geographical area as well as from other parts of India ${ }^{2}{ }^{2}$. Comparison of $10 \% \mathrm{KOH}$ mount and Gram stain showed sensitivities of $90.91 \%$ and $75 \%$ respectively. This correlates well with a similar study, which reported sensitivities of $99.23 \%$ and $88.75 \%$ for $10 \% \mathrm{KOH}$ mount and Gramsstained smear respectively ${ }^{13}$.

In the present study 14(31.81\%) samples remained sterile on culture though it was positive for fungal elements on direct microscopic examination. These were considered as positive because direct microscopic findings corroborated with the clinical findings of the patient. The reason for culture to remain sterile could be that the patients were already on topical antifungal agents before the corneal samples were taken. In our study, we could document 8 such cases who gave valid history of use of topical steroid use. Another reason could be that the sample was so scanty that only $\mathrm{KOH}$ wet mount preparation could be done and inadequate material was left for establishing cultures or the infiltration was deep stromal. The predominant fungus implicated in mycotic keratitis was Aspergillus species. This is in consonance with earlier reports. Aspergillus fumigatus is the commonest fungal agent reported from world wide, though Fusarium species and Candida albicans also been reported frequently ${ }^{10.14,15,16}$. In the present study Aspergillus fumigatus was the most common agent isolated followed by Aspergillus flavus, Aspergillus niger and Aspergillus glaucus.

Dematiaceous fungi, such as Bipolaris and Curvularia also have been reported as another common agent to cause keratitis $^{15,17,18}$. But in the present study no dematiaceous fungal agents were incriminated in keratitis. The present study also showed a wide spectrum of fungal aetiology of corneal ulcer.
The infectious keratitis is common in the 25-50yrs age group, which is the most susceptible group. Also it is found be more common in men (73.44\%) than in women ${ }^{2}$. In the present study the age of the patients ranged from 20 to 61 years and majority $(74.01 \%)$ of the cases were aged between 21-50years. However, the most susceptible age group was $41-50$ years (32.28\%) followed by $31-40$ years $(29.92 \%)$ and men were more commonly affected than women. This could be due to maximal outdoor activity of men, who have greater exposure to fungal agents.

Analysis of risk factors revealed corneal trauma with vegetative matter (54.54\%) as the commonest risk factor for mycotic keratitis, followed by use of topical antibacterial and/or steroid eye drops (18.18\%), and underlying chronic disease like diabetes mellitus. Easy availability of these topical agents and illiteracy often lead to prolonged use of these drugs, often beyond the prescribed period.

Seasonal variations and environmental factor such as humidity, rainfall, wind and harvesting of crops also influence incidence of mycotic keratitis. In our study, more number of cases (44.88\%) was reported in the months following rains, i: c September to December. Majority of the patients hailed from a rural background and most of them were involved in agriculture and animal husbandry.

\section{Conclusion:}

Mycotic keratitis continues to be an important cause of ocular morbidity, predominantly among rural population. Prompt diagnosis and early institution of antifungal therapy may limit the ocular morbidity and the sequelae of infectious keratitis. As the manifestation of mycotic keratitis is often confusing, a high degree of suspicion with sound knowledge of predisposing factors and microbiological confirmation is very essential to initiate appropriate therapy. 
Table 1: Co-rrelation between microscopy and culture findings.

\begin{tabular}{|l|c|c|c|c|}
\hline Investigation & Outcome & Number & \multicolumn{2}{|c|}{$\begin{array}{c}\text { Fungal growlth in culture } \\
\text { Positive }\end{array}$} \\
& & & Negative \\
\hline KOH wet mount & Positive & 40 & 22 & 18 \\
\hline & Negative & 04 & 04 & 00 \\
\hline & Total & 44 & 26 & 18 \\
\hline Gram's stain & Positive & 30 & 18 & 12 \\
\hline & Negative & 14 & 08 & 06 \\
\hline & Total & 44 & 26 & 18 \\
\hline
\end{tabular}

Table 2: Fungal isolates obtained on culture $(n=26)$

\begin{tabular}{|l|c|c|}
\hline $\begin{array}{l}\text { Isolate } \\
\text { I. Aspergillus species }\end{array}$ & No. & $\%$ \\
\hline a) A.fumigatus & 8 & $61.5 \%$ \\
\hline b) A.flavus & 4 & \\
\hline c) A.niger & 2 & \\
\hline d) A.glaucus & 1 & \\
\hline e) A.terreus & 1 & \\
\hline II. Fusarium species & 3 & $11.53 \%$ \\
\hline III. Penicillium species & 3 & $11.53 \%$ \\
\hline IV. Candida albicans & 2 & $7.69 \%$ \\
\hline V. Mucor & 1 & $3.86 \%$ \\
\hline VI. Paecilomyces & 1 & $3.86 \%$ \\
\hline
\end{tabular}

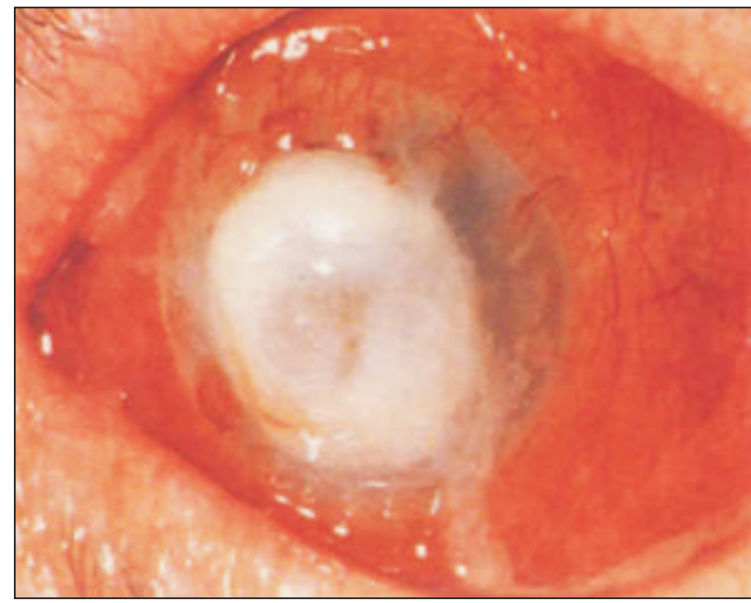

Figure 1: Infectious keratitis involving $360^{\circ}$ of limbus with impending perforation

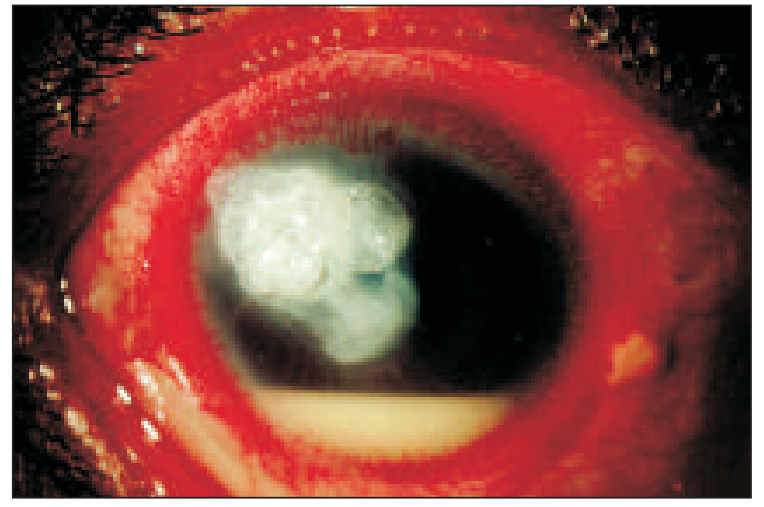

Figure 3: Aspergillus keratitis with tissue adhesive and bandage contact lensin place.
Table 3: Age and sex incidence of Mycotic keratitis $(n=44)$

\begin{tabular}{|l|c|c|c|}
\hline Age in years & Male & Female & Total \\
$21-30$ & 4 & 1 & 5 \\
\hline $31-40$ & 9 & 3 & 12 \\
\hline $41-50$ & 16 & 2 & 18 \\
\hline $51-60$ & 3 & 3 & 6 \\
\hline Above 60 & 2 & 1 & 3 \\
\hline Total & 34 & 10 & 44 \\
\hline
\end{tabular}

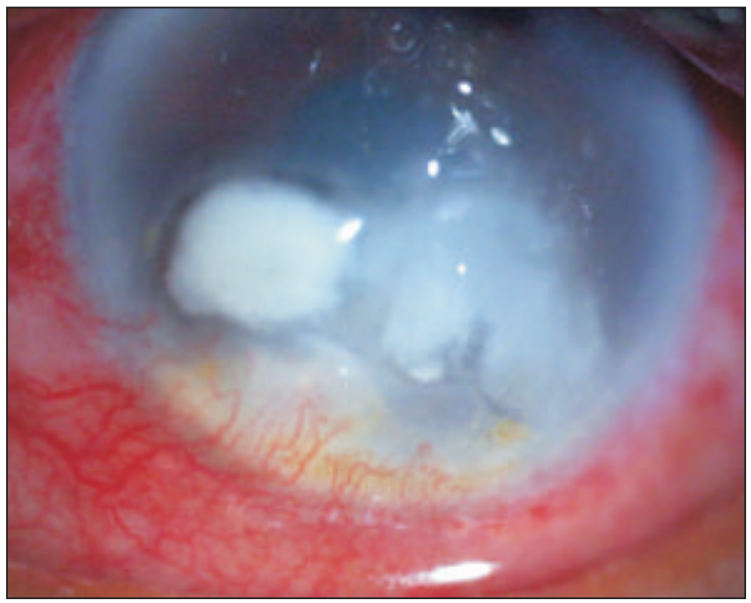

Figure 2: Fungal keratitis due to Candida albicans in pre existing corneal scar.

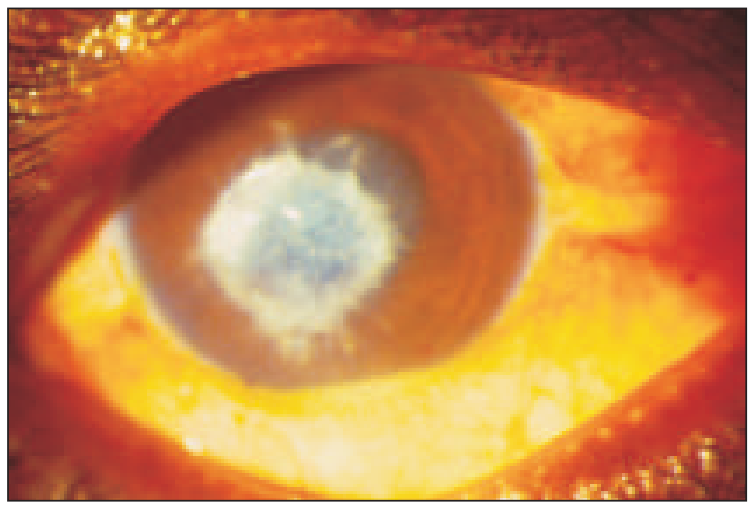

Figure 4: Fungal keratitis with classical feathery edges and satellite lesion. 


\section{References:}

1. Chirambo MC, Tielsch JM, West KP, Katz J. Blindness and visual important in southern Malian. Bull WHO 1986; 64: 567-72.

2. Bharathi MJ, Ramakrishnan R, Vasu S, M eenakshi R, Palaniappan $R$. Epimiological characteristics and laboratory diagnosis of fungal keratitis: a three year study. Indian J opthalmol 2003; 52:315-21.

3. Chander J. Oculomyces, Text book of Medical mycology, $2^{\text {nd }}$ Edn., Mehta Publishers: New Delhi 2002.

4. Kotigadde S, Ballal M, Jyothirlath AK. Mycotic keratitis: A Study in coastal Karnataka. Ind J Ophthalmol 1992; 40: 31-33.

5. Alfonso EC. Genotypic identification of Fusarium species from ocular sources: comparision to morphologic classification and antifungal sensitivity testing. TransAm Ophthalmol Soc 2008; 106:227-239.

6. Alfonso E, Mandelbaum S, Fox MJ, Forster RK. Ulcerative keratitis associated with contact lens wear. Am J Ophthalmol 1986; 101:429 433

7. Usha Arora, Parmjeet Kaur Gill, Samdeep Chalotra. Fungal Profile of Keratomycosis. Bombay Hospital Journal, Vol., No.3, 2009.

8. Sevel D, Kassar B. Suppurative keratitis and fungal keratitis. Trans Ophthalmol Soc NZ1973;25:228-232.

9. Chander J, Sinla N, Agnihotri N, Arya SK, Deep A. Keratomycosis in and around Chandigrah. A five year study from a North Indian Tertiary care hospital. Ind J Pathol M icrobiol 2008; 51(2) : 304-30.

10. Chander J, Sharma A. Prevalence of fungal corneal ulcers in Northern India. Infection 1994; 22:207-9.
11. Thomas PA. Current perspective on Ophthalmic mycoses. Clin Microbiol Rev 2003; 16: 730-97.

12. Bharathi M J, Ramakrishnan R, Vasu S, M eenakshi R, Palaniappan R. Aetiological diagnosis of microbial keratitis in south india: a study of 1618 cases. Indian J Med M icrobiol 2002; 19:19-24.

13. Srinivasan M. Fungal keratitis. Curr Opin Ophthalmol 2004; 15:321327.

14. Upadhyay MP, Karmacharya PC, Koirala S, Tuladhar NR, Bryan LE, Smolin G, Whitcher JP. Epidemiological characteristics of corneal ulceration in Nepal. AmJ Ophthalmol; 111:92-99.

15. Srinivasan M, Gonzales CA, George C, Cevallos V, Mascarenhas JM, Ashokan B, Wilkins J, Smolin G, Whitcher JP. Epidemiology and aetiological diagnosis of corneal ulceration in M adurai, South India. Br J Ophthalmol 1997; 81:965-71.

16. Rose Jr RH, M uller D, Alfonso EC. Changing spectrum of fungal keratitis in South Florida. Ophthalmology 1994; 101:1005-1013.

17. Gopinathan U, Fernandes S, Sharma S, Ananthan RGN. The epidemiological features and laboratory results of fungal keratitis: A 10 year review at a referral eye center in South India. Cornea 2002; 21:555-559.

18. Liesegang TJ, Forster RK., Spectrum of Microbial Keratitis in South Florida. Am J Ophthal 1980; 90:38-47 